\title{
Sistem Computer Vision Berbasis Metode Position Averaging Point untuk Pemilah Ikan Lele
}

\author{
Aditya Kurniawan, Kholilatul Wardani \\ Politeknik Kota Malang \\ Kompleks Pendidikan Internasional Tlogowaru No 3, telp/fax (0341) 754088 \\ e-mail: aditya@poltekom.ac.id
}

\begin{abstract}
Abstrak - Pada penelitian ini dibuat aplikasi machine vision yang menggunakan algoritma averaging point untuk mengatasi kesalahan pengukuran panjang ikan lele secara visual dikarenakan posisi tubuh (lengkungan) objek ukur yang bervariasi.algoritma ini bekerja dengan cara menentukan titik tengah, batas kiri dan kanan objek, serta menarik sebuah garis lurus yang melewati titik tengah untuk mengukur panjang objek.

Rata rata akurasi pengukuran dengan menggunakan algoritma ini adalah $96.5 \%$. Simpangan / error terbesar adalah $2.5 \mathrm{~mm}$ pada lengkungan dengan penyusutan dari ujung ke ujung sebesar $30 \%$. Berdasarkan perhitungan Pearson Correlation, disimpulkan bahwa lengkungan memiliki hubungan sebab akibat yang kecil terhadap error dalam pengukuran panjang ikan dengan konstanta pearson sebesar 0.26 .
\end{abstract}

Kata kunci: machine vision, averaging point, ikan lele, pearson

\section{Pendahuluan}

Machine Vision merupakan perkembangan dari aplikasi pengolahan citra (image processing) yang memiliki output berupa action dari aktuator. Image yang diidentifikasi oleh sensor / kamera akan dikirimkan ke komputer (yang dalam hal ini sebagai otak) yang kemudian diproses sedemikian rupa, selanjutnya komputer memberikan perintah kepada aktuator untuk melakukan sebuah perintah / aksi. Dengan machine vision, maka akan mempermudah proses produksi untuk menghasilkan benda/barang yang cepat dan reliabel. Dalam hal pemilah ikan lele yang memanfaatkan sistem computer vision untuk pemilahan ikan nya, didapatkan beberapa permasalahan diantaranya yaitu; (1) posisi ikan lele yang sedang diukur menggunakan algoritma image harus dalam posisi lurus. (2) posisi ikan lele yang melengkung akan mengurangi akurasi ukuran panjang ikan pada pengukuran barbasis image. Karena hal itulah maka perlu adanya pendekatan baru dalam sistem pemilah ikan berbasis computer vision yang dapat mengatasi permasalahan tersebut. Penelitian ini bertujuan untuk melihat bagaimana metode averaging point dapat meminimalisir kesalahan ukur panjang ikan yang berbentuk melengkung.

Algoritma yang digunakan pada penelitian ini adalah algoritma dasar feature extraction, yang mana pemisahan antara komponen warna (merah, hijau, dan biru) dipisahkan melalui algoritma sebagai berikut :

$$
\left.\left(R_{(x, y)}, G_{(x, y)}, B_{(x, y)}\right)=\left(\left\langle\mathrm{P}_{(x, y)} \operatorname{MOD}\left(2^{16}\right)\right\rangle \operatorname{MOD}\left(2^{8}\right)\right),\left(\operatorname{Int}\left\langle\frac{\left\langle\mathrm{P}_{(x, y)} \operatorname{MOD}\left(2^{16}\right)\right\rangle}{\left(2^{8}\right)}\right\rangle\right),\left(\operatorname{Int}\left\langle\frac{\mathrm{P}_{(x, y)}}{2^{16}}\right\rangle\right)\right)
$$

\footnotetext{
Dengan:

$\mathrm{P}(\mathrm{x}, \mathrm{y}) \quad$ : adalah nilai mentah pada pixel kolom y baris $\mathrm{x}$ yang bernilai 0 - 16581375

$\mathrm{R}(\mathrm{x}, \mathrm{y}) \quad$ : adalah nilai red (merah) pada pixel kolom y baris $\mathrm{x}$ yang bernilai $0-255$.

$\mathrm{G}(\mathrm{x}, \mathrm{y}) \quad$ : adalah nilai green (hijau) pada pixel kolom y baris $\mathrm{x}$ yang bernilai $0-255$.

$\mathrm{B}(\mathrm{x}, \mathrm{y}) \quad$ : adalah nilai blue (biru) pada pixel kolom y baris $\mathrm{x}$ yang bernilai 0-255.
}

TELKA, Vol.2, No.1, Mei 2016, pp. 10 17 


\section{Metodologi Penelitian}

Metode penelitian yang digunakan adalah dengan melakukan capture pada citra ikan lele dengan variasi lengkungan yang beragam, dan membandingkanya dengan ukuran lele yang sebenarnya (dalam $\mathrm{mm}$ ). Capture dengan menggunakan metode averaging ponit dilakukan sebanyak 10 kali capture pada panjang dan lengkungan ikan yang sama. Perulangan dilakukan dengan variasi lengkungan sebanyak 9 variasi. Setup penelitian dapat digambarkan seperti pada gambar Gambar1.

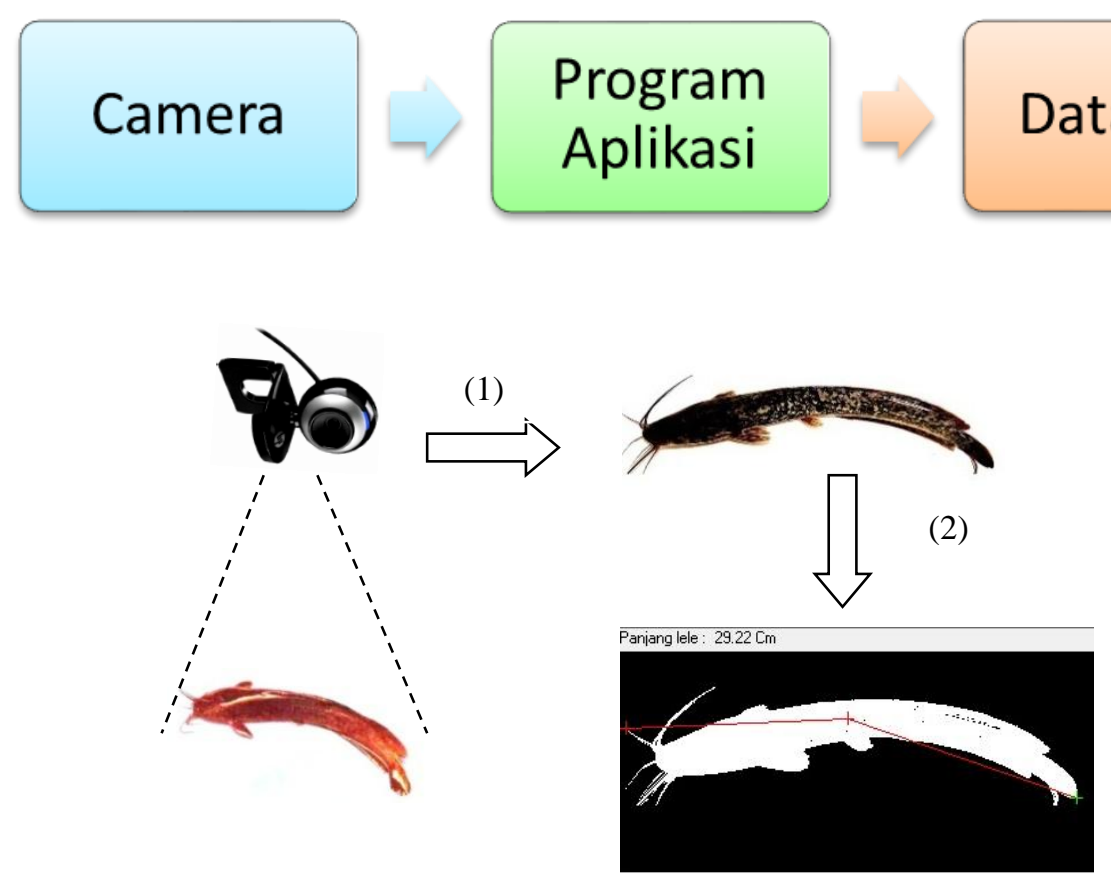

Gambar 1. Setup penelitian

Tabel 1. Variabel penelitian

\begin{tabular}{|c|c|}
\hline $\begin{array}{c}\text { Variabel } \\
\text { independen }\end{array}$ & $\begin{array}{c}\text { Variabel } \\
\text { dependen }\end{array}$ \\
\hline Panjang lele & Pixel \\
\hline
\end{tabular}

Tabel 2. Variabel penelitian

\begin{tabular}{|c|c|c|}
\hline No & Panjang lele & Lengkungan \\
\hline 1 & $40 \mathrm{~mm}$ & $90 \%$ \\
\hline 2 & $40 \mathrm{~mm}$ & $80 \%$ \\
\hline 3 & $40 \mathrm{~mm}$ & $70 \%$ \\
\hline 4 & $40 \mathrm{~mm}$ & $60 \%$ \\
\hline 5 & $40 \mathrm{~mm}$ & $50 \%$ \\
\hline 6 & $40 \mathrm{~mm}$ & $40 \%$ \\
\hline 7 & $40 \mathrm{~mm}$ & $30 \%$ \\
\hline 8 & $40 \mathrm{~mm}$ & $20 \%$ \\
\hline 9 & $40 \mathrm{~mm}$ & $10 \%$ \\
\hline
\end{tabular}




\section{Hasil dan Pembahasan}

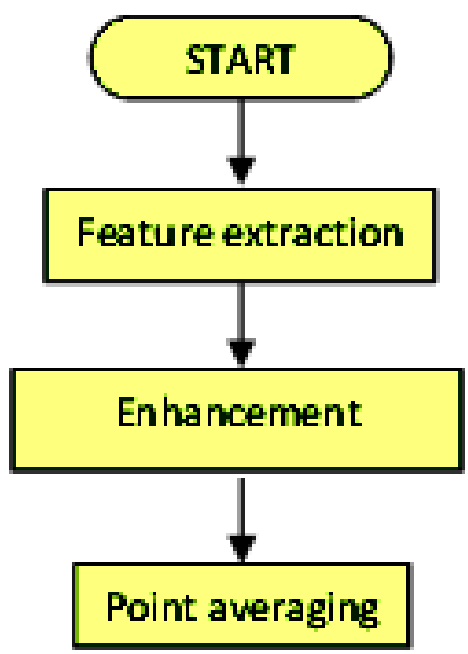

Gambar 2. flowchart sistem

Pada penelitian ini digunakan proses algoritma yang memiliki 3 langkah yaitu feature extraction, image enhancement, dan point averaging. Dalam proses feature extraction, data mentah dalam pixel $\mathrm{x}, \mathrm{y}$ di ekstraksi menjadi 3 komponen warna yaitu $\mathrm{R}(\mathrm{x}, \mathrm{y}) ; \mathrm{G}(\mathrm{x}, \mathrm{y})$ dan $\mathrm{B}(\mathrm{x}, \mathrm{y})$ dengan rentang nilai antara 0-255. Algoritma yang digunakan adalah sebagai berikut:

$\left.\left(R_{(x, y)}, G_{(x, y)}, B_{(x, y)}\right)=\left(\left\langle\mathrm{P}_{(x, y)} \operatorname{MOD}\left(2^{16}\right)\right\rangle \operatorname{MOD}\left(2^{8}\right)\right),\left(\operatorname{Int}\left\langle\frac{\left\langle\mathrm{P}_{(x, y)} \operatorname{MOD}\left(2^{16}\right)\right\rangle}{\left(2^{8}\right)}\right\rangle\right),\left(\operatorname{Int}\left\langle\frac{\mathrm{P}_{(x, y)}}{2^{16}}\right\rangle\right)\right)$

Listing program yang digunakan adalah dengan membuat function sebagai berikut :

Function BacaDanEkstrakAll(indexSimpanDataOutput As Integer, pictureboxYangAkanDibaca As PictureBox) As String

For $\mathrm{i}=1$ To pictureboxYangAkanDibaca.ScaleWidth

For $\mathrm{j}=1$ To pictureboxYangAkanDibaca.ScaleHeight

Pixel(indexSimpanDataOutput, $\mathrm{i}, \mathrm{j})=$ pictureboxYangAkanDibaca.Point $(\mathrm{i}, \mathrm{j})$

If Pixel(indexSimpanDataOutput, $\mathrm{i}, \mathrm{j}$ ) $<0$ Then Pixel(indexSimpanData, $\mathrm{i}, \mathrm{j})=0$

Next j

Next i

For $\mathrm{i}=1$ To pictureboxYangAkanDibaca.ScaleWidth

For $\mathrm{j}=1$ To pictureboxYangAkanDibaca.ScaleHeight

RED(indexSimpanDataOutput, $i, j)=\operatorname{Int}(($ Pixel(indexSimpanDataOutput, $i, j) \operatorname{Mod}(2 \wedge(2 * 8))) \operatorname{Mod}(2 \wedge 8))$

GREEN(indexSimpanDataOutput, $i, j)=\operatorname{Int}(($ Pixel(indexSimpanDataOutput, $i, j) \operatorname{Mod}(2 \wedge(2 * 8))) /(2 \wedge 8))$

BLUE(indexSimpanDataOutput, $i, j)=\operatorname{Int}(($ Pixel(indexSimpanDataOutput, $i, j)) /(2 \wedge(2 * 8)))$

Next j

Next i

End Function

Setelah itu, proses enhancement yaitu brightness dan contrast yang digunakan untuk memisahkan objek (ikan) dengan background warna dengan menggunakan algoritma berikut: 


$$
\text { Rbright }_{(x y y)}, \text { Gbright }_{(x y),} \text { Bright }_{(x y y)}=R_{(x, y)}+C b, G_{(x, y)}+C b, B_{(x, y)}+C b
$$

Dengan :

$\operatorname{Rbright}(\mathrm{x}, \mathrm{y})$, Gbright $(\mathrm{x}, \mathrm{y})$, Bbright $(\mathrm{x}, \mathrm{y})=$ adalah nilai komponen warna pada pixel kolom y baris $\mathrm{x}$ setelah operasi pencerahan / brightness

Setelah itu, proses contrast sebagai proses enhancement final yang bertujuan untuk memisahkan objek dan background dengan menggunakan algoritma berikut :

$$
\operatorname{Rcont}_{(x, y)}, G \operatorname{cont} t_{(x y)}, B \operatorname{cont} t_{(x y y)}=\left(R_{(x, y)}+C b\right) x C c_{,}\left(G_{(x, y)}+C b\right) x C c_{,}\left(B_{(x, y)}+C b\right) x C c
$$

Dengan :

$\operatorname{Rcont}(\mathrm{x}, \mathrm{y})$, Gcont $(\mathrm{x}, \mathrm{y}), \mathrm{Bcont}(\mathrm{x}, \mathrm{y})=$ adalah nilai komponen warna pada pixel kolom y baris $\mathrm{x}$ setelah operasi pencerahan / brightness dan contrast

$\mathrm{Cc}=$ adalah contanta contrast yang bernilai $0.1-1.9$

Listing program yang digunakan adalah dengan membuat function sebagai berikut :

Listing program brightness

Function PointProcessingBrightnessAll(IndexSimpanDataInput As Integer, indexSimpanDataOutput As Integer, pictureboxYangAkanDiproses As PictureBox, ContsBrightness As Integer)

For $\mathrm{i}=1$ To pictureboxYangAkanDiproses.ScaleWidth

For $\mathrm{j}=1$ To pictureboxYangAkanDiproses.ScaleHeight

RED(indexSimpanDataOutput, $i, j$ ) = RED(IndexSimpanDataInput, $i, j$ ) + ContsBrightness

GREEN(indexSimpanDataOutput, $i, j$ ) = GREEN(IndexSimpanDataInput, $i, j$ ) + ContsBrightness

BLUE(indexSimpanDataOutput, $i, j$ ) = BLUE(IndexSimpanDataInput, $i, j$ ) + ContsBrightness

Next j

Next i

End Function

Listing program contrast

Function PointProcessingContrastAll(IndexSimpanDataInput As Integer, indexSimpanDataOutput As Integer, pictureboxYangAkanDiproses As PictureBox, ContsContrast10sd190 As Double)

Dim ContsContrast As Double

ContsContrast $=$ ContsContrast10sd190 $/ 100$

For $\mathrm{i}=1$ To pictureboxYangAkanDiproses.ScaleWidth

For $\mathrm{j}=1$ To pictureboxYangAkanDiproses.ScaleHeight

RED(indexSimpanDataOutput, $i, j$ ) = RED(IndexSimpanDataInput, $i, j$ ) * ContsContrast

GREEN(indexSimpanDataOutput, i, j) = GREEN(IndexSimpanDataInput, i, j) * ContsContrast

BLUE(indexSimpanDataOutput, $\mathrm{i}, \mathrm{j}$ ) = BLUE(IndexSimpanDataInput, $\mathrm{i}, \mathrm{j}) *$ ContsContrast

Next j

Next i

End Function

Setelah melewati proses enhancement, maka proses selanjutnya adalah colour filtering untuk memisahkan background dengan objek. Perhitungan ini dilakukan dengan teknik sebagai berikut: 


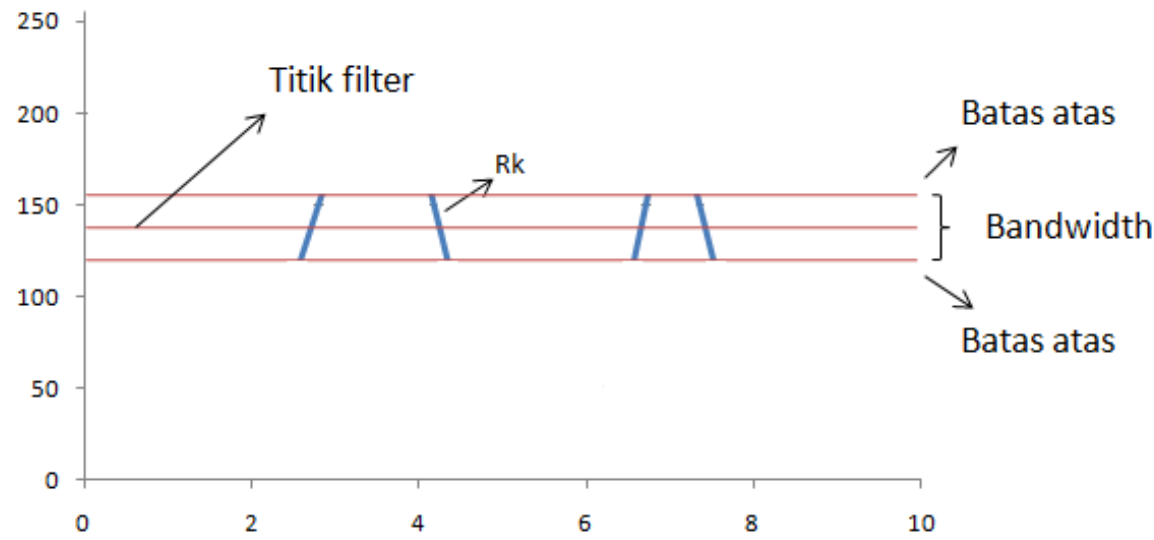

Gambar 3. grafik colour filtering

Listing program yang digunakan adalah dengan membuat function sebagai berikut:

Function FilterWarnaMonotoneThresholdingHasilPutihResiduHitam(IndexSimpanDataInput As Integer, indexSimpanDataOutput As Integer, pictureboxYangAkanDiproses As PictureBox, Rthres As Integer, Gthres As Integer, Bthres As Integer, Colourband As Integer)

For $\mathrm{i}=1$ To pictureboxYangAkanDiproses.ScaleWidth

For $\mathrm{j}=1$ To pictureboxYangAkanDiproses.ScaleHeight

If RED(IndexSimpanDataInput, i, j) > (Rthres - Colourband / 2) And RED(IndexSimpanDataInput, i, j) < (Rthres + Colourband / 2) And GREEN(IndexSimpanDataInput, i, j) > (Gthres - Colourband / 2) And GREEN(IndexSimpanDataInput, i, j) < (Gthres + Colourband / 2) And BLUE(IndexSimpanDataInput, i, j) > (Bthres Colourband / 2) And BLUE(IndexSimpanDataInput, i, j) < (Bthres + Colourband / 2) Then

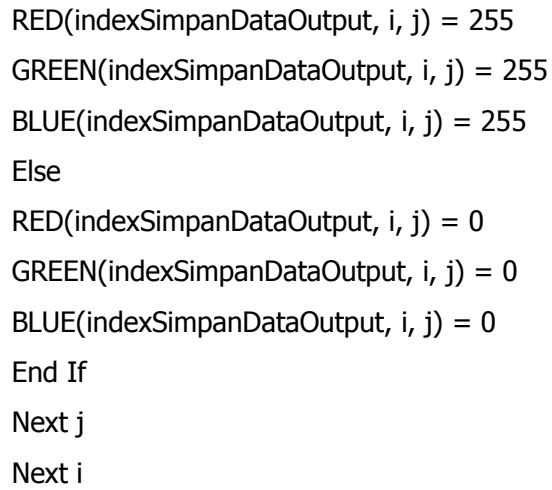

Point averaging adalah sebuah proses yang dapat memberikan koordinat titik tengah objek dengan melakukan perhitungan averaging (rata rata) pada pixel yang berkaitan dengan objek untuk menentukan koordinat tengah yang tepat. Perhitungan ini dilakukan dengan algoritma awal sebagai berikut:

Untuk $\mathrm{x}=0$

If $R_{(0, y)} A N D G_{(0, y)} A N D B_{(0, y)}=0$ then $\mathrm{X}(0)=\mathrm{X}(0)+1$ 
Untuk y $=0$

If $R_{\left(x_{2}, 0\right)} A N D G_{\left(x_{0}, 0\right)} A N D B_{\left(x_{2}, 0\right)}=0$ then $\mathrm{Y}(0)=\mathrm{Y}(0)+1$

Maka dari itu akan didapatkan $\mathrm{X}(0)-\mathrm{X}(\mathrm{pW})$ dan $\mathrm{Y}(0)-\mathrm{Y}(\mathrm{pH})$

Dengan :

$\mathrm{pW}=$ Picture width

$\mathrm{pH}=$ Picture height

Untuk mendapatkan titik tengah untuk masing masing koordinat, maka digunakan algoritma :

$$
X=\frac{\sum_{i=0}^{p W} X(i)}{T p o}, Y=\frac{\sum_{i=0}^{p H} Y(i)}{T p o}
$$

Dengan :

Tpo $=$ Total pixel objek

Listing program yang digunakan adalah dengan membuat function sebagai berikut :

Function HitungCentreObject(IndexSimpanDataInput As Integer, pictureboxYangAkanDiproses As PictureBox)

Dim HitungPixelPutihH, HitungPixelPutihV As Double

HitungPixelPutihH $=0$

HitungPixelPutihV $=0$

For $\mathrm{i}=1$ To pictureboxYangAkanDiproses.ScaleWidth -1

For $\mathrm{j}=1$ To pictureboxYangAkanDiproses.ScaleHeight -1

If RED(IndexSimpanDataInput, $\mathrm{i}, \mathrm{j}$ ) = 255 Then HitungPixelPutihH = HitungPixelPutihH $+\mathrm{i}$

Next j

Next $\mathrm{i}$

For $\mathrm{j}=1$ To pictureboxYangAkanDiproses.ScaleWidth - 1

For $\mathrm{i}=1$ To pictureboxYangAkanDiproses.ScaleHeight - 1

If RED(IndexSimpanDataInput, $i, j$ ) $=255$ Then HitungPixelPutihV $=$ HitungPixelPutihV $+j$

Next i

Next j

HitungCentreObjectHc $=$ Int(HitungPixelPutihH / (HitungPixelPutih(IndexSimpanDataInput, pictureboxYangAkanDiproses)))

HitungCentreObjectVc = Int(HitungPixelPutihV / (HitungPixelPutih(IndexSimpanDataInput, pictureboxYangAkanDiproses)))

End Function 
Penelitian ini menghasilkan data sebagai berikut :

Tabel 3. Variabel penelitian

\begin{tabular}{|c|c|c|c|c|c|}
\hline No & $\begin{array}{c}\text { Panjang ikan } \\
\text { (sebenarnya) }\end{array}$ & Lengkungan & Pixel & $\begin{array}{c}\text { Panjang ikan } \\
\text { (terukur) }\end{array}$ & $\begin{array}{c}\text { error } \\
\text { (dalam \%) }\end{array}$ \\
\hline 1 & $40 \mathrm{~mm}$ & $90 \%$ & 234 & $39 \mathrm{~mm}$ & 2.5 \\
\hline 2 & $40 \mathrm{~mm}$ & $80 \%$ & 226 & $37.6 \mathrm{~mm}$ & 6 \\
\hline 3 & $40 \mathrm{~mm}$ & $70 \%$ & 237 & $39.4 \mathrm{~mm}$ & 1.5 \\
\hline 4 & $40 \mathrm{~mm}$ & $60 \%$ & 227 & $37.8 \mathrm{~mm}$ & 5.5 \\
\hline 5 & $40 \mathrm{~mm}$ & $50 \%$ & 237 & $39.5 \mathrm{~mm}$ & 1.25 \\
\hline 6 & $40 \mathrm{~mm}$ & $40 \%$ & 234 & $38.9 \mathrm{~mm}$ & 2.75 \\
\hline 7 & $40 \mathrm{~mm}$ & $30 \%$ & 225 & $37.5 \mathrm{~mm}$ & 6.25 \\
\hline 8 & $40 \mathrm{~mm}$ & $20 \%$ & 235 & $39.1 \mathrm{~mm}$ & 2.25 \\
\hline 9 & $40 \mathrm{~mm}$ & $10 \%$ & 239 & $39.8 \mathrm{~mm}$ & 0.5 \\
\hline
\end{tabular}

$\begin{array}{ll}\text { Maksimum panjang terukur } & : 39.8 \mathrm{~mm} \\ \text { Minimum panjang terukur } & : 37.5 \mathrm{~mm} \\ \text { Maksimum error } & : 6.25 \% \\ \text { Minimum error } & : 0.5 \% \\ \text { Rata rata panjang terukur } & : 38.7 \mathrm{~mm} \\ \text { Rata rata error } & : 3.1 \%\end{array}$

Dengan menggunakan pearson correlation coefficient dengan membandingkan variabel lengkungan ikan dengan error pengukuran maka didapatkan :

$$
r=\frac{\sum_{i=1}^{n}\left(X_{i}-\bar{X}\right)\left(Y_{i}-\bar{Y}\right)}{\sqrt{\sum_{i=1}^{n}\left(X_{i}-\bar{X}\right)^{2}} \sqrt{\sum_{i=1}^{n}\left(Y_{i}-\bar{Y}\right)^{2}}} .
$$

Dengan

variable $\mathrm{X}=$ lengkungan ikan

variable $\mathrm{Y}=$ error pengukuran

maka didapatkan

$\mathrm{r}=0.26$

Tabel 4. pearson coefficient

\begin{tabular}{|c|c|c|}
\hline Correlation & Negative & Positive \\
\hline None & -0.09 to 0.0 & 0.0 to 0.09 \\
\hline Small & -0.3 to -0.1 & 0.1 to 0.3 \\
\hline Medium & -0.5 to -0.3 & 0.3 to 0.5 \\
\hline Large & -1.0 to -0.5 & 0.5 to 1.0 \\
\hline
\end{tabular}

\section{Kesimpulan}

Algoritma averaging point dapat mendeteksi ukuran panjang ikan lele dengan akurasi rata rata sebesar $96.9 \%$. Simpangan / error terbesar adalah $2.5 \mathrm{~mm}$ pada lengkungan dengan 
penyusutan dari ujung ke ujung sebesar 30\%. Berdasarkan perhitungan Pearson Correlation, dapat disimpulkan bahwa lengkungan memiliki hubungan sebab akibat yang kecil terhadap error dalam pengukuran panjang ikan (konstanta $=0.26$ ). hal ini mengindikasikan algoritma averaging point relatif baik dalam melakukan pengukuran terhadap ikan dengan posisi yang bervariasi dibandingkan dengan tanpa menggunakan algoritma ini.

Penelitian ini secara keseluruhan belum mendalami tentang variasi iluminasi yang memeiliki tingkat kemungkinan tinggi dalam kemunculan error pengukuran panjang ikan. Pada penilitian selanjutnya diharapkan dapat mendalami hubungan tingkat iluminasi dengan tingkat error pengukuran.

\section{Daftar Pustaka}

[1] Lim, Kelvin K. P. Clarias batu, a New Species of Catfish (Teleostei: Claridae) from Pulau Tioman, Peninsular Malaysia. The Raffles Bulletin of Zoology. Malaysia. 1999; 6: 157-167

[2] Misimi, E., Mathiassen., Erikson, U. Computer Vision - Based Sorting of Atlantic Salmon (Salmo salar) Fillets According to Their Color Level. Journal of Food Science. -. 2007; 72:1

[3] Ng, Heok Hee. Clarias insolitus, a new species of clariid catfish (Teleostei: Siluriformes) from southern Borneo. Zootaxa. -. 2003; 284:1-8

[4] Sudarto, Teugels, Guy G, Pouyaud, Laurent. Description of a New Clariid Catfish, Clarias pseudonieuhofii from West Borneo (Siluriformes: Clariidae). Zoological Studies. -. 2004; 43:8-19

[5] White, D.J. Automated Measurement of Species and Length of Fish by Computer Vision. University of Aberdeen Journal. United Kingdom. 2006;

[6] Kenneth R, Castleman. Digital Image Processing. 1st. - : Prentice Hall. 1996: 245. 